\title{
Acousto-Electric Interaction in Magnetised Piezoelectric Semiconductor Quantum Plasma
}

\author{
S. Ghosh And A. Muley* \\ School of Studies in Physics, Vikram University, Ujjain 456010, India \\ (Received March 29, 2016; in final form August 31, 2016)
}

\begin{abstract}
Amplification of an acoustic wave is considered in magnetised piezoelectric $n$-type semiconductor plasma under quantum hydrodynamic regime. The important ingredients of this study are the inclusion of quantum diffraction effect via the Bohm potential, statistical degeneracy pressure, and externally applied magnetostatic field in the momentum balance equation of the charged carriers. A modified dispersion relation is derived for evolution of acoustic wave by employing the linearization technique. Detailed analysis of quantum modified dispersion relation of acoustic wave is presented. For a typical parameter range, relevant to $n$-InSb at $77 \mathrm{~K}$, it is found that the non-dimensional quantum parameter $H$ reduces the gain while magnetic field enhances the gain of acoustic wave. The crossover from attenuation to amplification occurs at $\left(\vartheta_{0} / \vartheta_{s}\right)=1$ and this crossover point is found to be unaffected by quantum correction and magnetic field. It is also found that the maximum gain point shifts towards lower drift velocity regime due to the presence of magnetic field while quantum parameter $H$ shifts this point towards higher drift velocity. Numerical results on the acoustic gain per radian and acoustic gain per unit length are also illustrated. Our results could be useful in understanding acoustic wave propagation in magnetised piezoelectric semiconductor in quantum regime.
\end{abstract}

DOI: 10.12693/APhysPolA.130.1401

PACS/topics: 72.50.+b, 52.35.-g, 61.72.uj, 77.65.-j

\section{Introduction}

The field of quantum plasma physics [1-5] has considerable interest having risen recently in connection with its potential applications in modern technology, in emerging miniaturization techniques. In quantum plasmas, due to inter-fermion distances much lower than its de Broglie wavelength and the influence of the Pauli exclusion rule, many quantum effects such as electron-tunnelling, degeneracy pressure and Landau quantization may occur [6-8]. The outstanding deviations in linear and nonlinear features of quantum plasmas from that classical ones [9-12] were reported. Most of these works are based on quantum hydrodynamic (QHD) model of plasmas. This model is very useful to study the short-scale collective phenomena, such as waves, instabilities, linear and nonlinear interactions in dense plasmas [13-15]. By the inclusion of the statistical degeneracy pressure and quantum diffraction (known as the Bohm potential) terms, QHD model becomes a generalization of the usual fluid model. A form of QHD model has also been proposed incorporating a non-dimensional quantum term $H$, which is reminiscent of quantum diffraction and Fermi degenerate pressure, and is defined as ratio of plasmon, an elementary excitation associated with electron plasma wave, and the Fermi energy of the system. The validity of QHD model is limited to those systems, that are large compared to the Fermi lengths of the species in the system.

A possible application of quantum plasma also occurs in some semiconductor materials [16-20], the miniaturization of electronic components made up of

\footnotetext{
*corresponding author; e-mail: apurva.muley.a@gmail.com
}

semiconductors is based on the fact that the de Broglie wavelength of charge carriers in these media can be made comparable, to the spatial variation of the doping profiles. Hence, typical quantum mechanical effects are expected to play a central role in the behaviour of electronic components, to be constructed in near future. When electrons from valence band of the semiconductor are excited to the conduction band, the system can be considered as a plasma medium that satisfies the plasma conditions. The linear and nonlinear behaviours of waves and instabilities, through the carrier's dynamics in semiconductors, are crucially important for modern device physicists dealing with quantum wells, quantum wires, quantum dots, etc. In the development of wireless electronic devices, wireless power sources have become an important issue. Piezoelectric materials are usually used to convert electrical energy into mechanical energy or vice versa, and hence, have been receiving broad and sustained attention. Piezoelectric semiconductors have been used to make devices for acoustic wave amplification [21-25], and acoustic charge transport based on the motion of carriers under the electric field accompanying an acoustic wave.

Significant research efforts have been devoted to acoustic wave amplification in piezoelectric semiconductor plasma medium [26-28], but at present, there is very little understanding of effects of the Bohm potential and statistical degeneracy pressure on acousto-electric interactions, and resulted acoustic amplification in piezoelectric semiconductor plasma, particularly in presence of magnetic field. Hence, in this paper, we have presented the effects of these quantum correction terms and magnetostatic field on dispersion and gain profiles of acoustic wave, excited within a piezoelectric semiconductor plasma medium, using QHD model of plasma. 


\section{Theoretical formulation}

In the present model, we consider homogeneous piezoelectric semiconductor quantum plasma of group III-V, in which electrons are majority charge carriers. Let the medium be immersed in a dc electric field $\boldsymbol{E}_{0}$, which is applied along $z$-axis, and magnetic field $\boldsymbol{B}_{0}$ applied in the $x-z$ plane, making an arbitrary angle $\theta$ with the $z$-axis. In the present paper, we have analysed the role of magnetic field under quantum regimes on the acoustic wave propagation, which is taken to be propagating along $z$ axis. A linear dispersion relation for acoustic wave may be obtained by using fluid equations of QHD model. The relevant equations of QHD model (i.e. continuity and momentum transfer equation), in terms of non-dimensional quantum parameter $H$, may be described as follows:

$$
\begin{aligned}
& \frac{\partial n_{1}}{\partial t}+n_{0}\left(\nabla \cdot \boldsymbol{\vartheta}_{1}\right)+\boldsymbol{\vartheta}_{0}\left(\nabla \cdot n_{1}\right)=0, \\
& \left(\frac{\partial \boldsymbol{\vartheta}_{1}}{\partial t}+\boldsymbol{\vartheta}_{0} \cdot \nabla \boldsymbol{\vartheta}_{1}\right)+\nu \boldsymbol{\vartheta}_{1}+\boldsymbol{\vartheta}_{1} \times \boldsymbol{\omega}_{c}= \\
& \frac{-e}{m}\left(\boldsymbol{E}_{1}\right)-\vartheta_{\mathrm{F}}^{2}\left(1+\frac{k^{2} \vartheta_{\mathrm{F}}^{2}}{4 \omega_{p}^{2}} H^{2}\right) \frac{\nabla n_{1}}{n_{0}} .
\end{aligned}
$$

The momentum transfer equation of QHD model differs from that of classical hydrodynamic model, through the presence of the last term in Eq. (2). This term adequately serves the effects of quantum diffraction and Fermi degenerate pressure in plasma system.

Here $H=\hbar \omega_{p} / 2 k_{\mathrm{B}} T_{\mathrm{F}}$ is the non-dimensional quantum parameter, measuring the relevance of quantum effects, and is proportional to quantum diffraction. $\vartheta_{0}$ is drift velocity of the carriers due to applied electrostatic field $\boldsymbol{E}_{0}$, $\vartheta_{1}$ is the perturbed velocity, $(-e)$ and $m$ are the charge and mass of an electron, $n_{0}$ and $n_{1}$ are the equilibrium and perturbed electron number density, $\nu$ is the momentum transfer collision frequency, $\vartheta_{\mathrm{F}}=\left(2 k_{\mathrm{B}} T_{\mathrm{F}} / m\right)^{1 / 2}$ is Fermi velocity at Fermi temperature $T_{\mathrm{F}}, \omega_{c}=e B_{0} / m$ is electron cyclotron frequency due to applied magnetic field $\boldsymbol{B}_{0}$ and $\omega_{p}=\sqrt{e^{2} n_{0} / m \varepsilon}$ is the electron plasma frequency; $\varepsilon=\varepsilon_{0} \varepsilon_{L}$ ) being permittivity of the medium with lattice dielectric constant $\varepsilon_{L}$.

We assume that all the small wave perturbations vary as $\exp (\mathrm{i}(\omega t-k z))$ and travelling with phase velocity $(\omega / k)$. Here $\omega$ and $k$ are the wave angular frequency and wave number, respectively. Under the considered field geometry, the wave equation in an elastic piezoelectric medium becomes

$$
\left(-\rho \omega^{2}+C k^{2}\right) u_{x}=\mathrm{i} k \bar{e} E_{z} .
$$

Here $C$ and $\bar{e}$ are coefficient of elastic stiffness and piezoelectricity of the medium and all other symbols have the same meaning as defined by Steele and Vural [29].

Using Eqs. (1), (2), the component of perturbed velocity of electrons along propagation direction comes out as

$$
\vartheta_{1 z}=\frac{\mathrm{i}(e / m)}{F_{Q}(\omega, k)} E_{z}
$$

where

$$
\begin{aligned}
& F_{Q}(\omega, k)=\left(\omega-k \vartheta_{0}-\mathrm{i} \nu-\frac{\nu D_{\mathrm{F}} k^{2}\left(1+\frac{k^{2} \vartheta_{\mathrm{F}}^{2}}{4 \omega_{p}^{2}} H^{2}\right)}{\omega-k \vartheta_{0}}\right) \\
& +\frac{\omega_{c}^{2} \sin ^{2} \theta\left(\omega-k \vartheta_{0}-\mathrm{i} \nu\right)}{\omega_{c}^{2} \cos ^{2} \theta-\left(\omega-k \vartheta_{0}-\mathrm{i} \nu\right)^{2}} .
\end{aligned}
$$

Here $D_{\mathrm{F}}=\vartheta_{\mathrm{F}}^{2} / \nu$ is diffusion constant at the Fermi temperature.

Following Steele and Vural [29] and White [30], and using Eqs. (1)-(4), we get the quantum modified dispersion relation for acousto-electric interactions in magnetised piezoelectric semiconductor quantum plasma as

$$
\left(\omega^{2}-k^{2} \vartheta_{s}^{2}\right)\left(1-\frac{\omega_{p}^{2}}{\left(\omega-k \vartheta_{0}\right) F_{Q}(\omega, k)}\right)=K^{2} k^{2} \vartheta_{s}^{2} .
$$

In Eq. (5), term on right hand side (R.H.S.) represents the coupling between phonon and plasmon with $K^{2}=\bar{e}^{2} / \varepsilon C$, the dimensionless electro-mechanical coupling constant, and $\vartheta_{s}=\sqrt{C / \rho}$ the acoustic velocity in the medium. As evident from Eq. (5), this coupling parameter couples two independent modes, expressed as

$$
\begin{aligned}
& \omega^{2}-k^{2} \vartheta_{s}^{2}=0, \\
& \left(1-\frac{\omega_{p}^{2}}{\left(\omega-k \vartheta_{0}\right) F_{Q}(\omega, k)}\right)=0 .
\end{aligned}
$$

Equation (6) denotes usual sound mode, propagating through an elastic medium with velocity $\vartheta_{s}$, and Eq. (7) represents the modified electro-kinetic mode in presence of magnetic field and quantum parameter $H$.

Now using the approximation $\frac{k \vartheta_{s}}{\omega}=1+\mathrm{i} \alpha$ [30], where the gain per radian $\alpha \ll 1$, in the collision dominated regime $\left(\omega, k \vartheta_{0} \ll \nu\right)$, Eq. (5) reduces to

$$
\alpha=\frac{\frac{1}{2} K^{2} \gamma \omega_{R} / \omega \varphi}{\left(\frac{\omega_{R}}{\omega \varphi}\right)^{2}\left(1+\frac{\omega^{2}\left(1+\frac{k^{2} \vartheta_{\mathrm{F}}^{2}}{4 \omega_{p}^{2}} H^{2}\right)}{\omega_{R} \omega_{D F}}\right)^{2}+\gamma^{2}} .
$$

Here $\omega_{R}=\omega_{p}^{2} / \nu$ is dielectric relaxation frequency, $\omega_{D F}=$ $\vartheta_{s}^{2} / D_{\mathrm{F}}$ is diffusion frequency at the Fermi temperature, $\gamma=\frac{\vartheta_{0}}{\vartheta_{s}}-1$ and $\varphi=\frac{1+\omega_{c}^{2} / \nu^{2}}{1+\omega_{c}^{2} \cos ^{2} \theta / \nu^{2}}$. The above expression represents gain coefficient in terms of quantum parameter $H$, and magnetic parameter $\varphi$. At $\alpha>0$ i.e. when gain coefficient becomes positive, one obtains the amplification of acoustic wave. This condition $(\alpha>0)$ for acoustic gain can be achieved from Eq. (8), only when $\gamma>0$ or $\frac{\vartheta_{0}}{\vartheta_{s}}>1$. It is also clear from Eq. (8), that the signature of magnetic field appears through the parameter $\varphi$, and that of quantum correction through parameter $H$.

For classical plasma medium $(H \rightarrow 0)$, this dispersion relation (Eq. (8)) reduces to equation (8-44) of Steele and Vural [29]. Hence, this quantum modified dispersion relation is expected to give modified results, compared to that obtained for classical plasma.

\section{Results and discussion}

In this section, we analyze the quantum modified acoustic wave dispersion relation in presence of magnetic 
field. For the numerical appreciation of our results, we consider the following parameters of a moderately piezoelectric $n$-InSb medium at $77 \mathrm{~K}$. The momentum transfer collision frequency $\nu=3.5 \times 10^{11} \mathrm{~s}^{-1}$, piezoelectric constant $\bar{e}=0.054 \mathrm{C} \mathrm{m}^{-2}$, mass density $\rho=$ $5.8 \times 10^{3} \mathrm{~kg} \mathrm{~m}^{-3}$, electron effective mass $m=0.014 m_{0}$ with $m_{0}$ being free electron mass and lattice dielectric constant $\varepsilon_{L}=17.54$.

Depending on the above physical constants, we have investigated the acoustic gain behaviour by varying cyclotron frequency $\omega_{c}$, for different magnitude of quantum parameter $H$, and also by varying the ratio of drift to sound velocities $\frac{\vartheta_{0}}{\vartheta_{s}}$, for four different combinations of magnitude of quantum parameter $H$, and magnetic field $B_{0}$. We also represent the influence of drifting of electrons $\left(\vartheta_{0}\right)$ on gain per unit length $\left(\frac{\alpha \omega}{\vartheta_{s}}\right)$ in presence and absence of magnetostatic field $B_{0}$. All these results are displayed in Figs. 1-3.

The variation of gain per unit length $\frac{\alpha \omega}{\vartheta_{s}}$, with magnetic field through cyclotron frequency $\omega_{c}$, using quantum parameter $H$ as parameter, is depicted in Fig. 1. The nature of variation is identical for all values of $H$ i.e. $\frac{\alpha \omega}{\vartheta_{s}}$ increases with increasing value of $\omega_{c}$, and then nearly saturates towards higher cyclotron frequency regime $\left(\omega_{c} \gg \nu\right)$. From this figure, it is found that application of magnetic field enhances the magnitude of acoustic gain. Thus the magnetostatic field increases the tendency of a stable acoustic wave to become unstable, and in case of instability, it increases the gain. It is also evident from the curves of this figure that the gain per unit length shrinks on increasing the quantum diffraction term $H$, at a constant magnetic field.

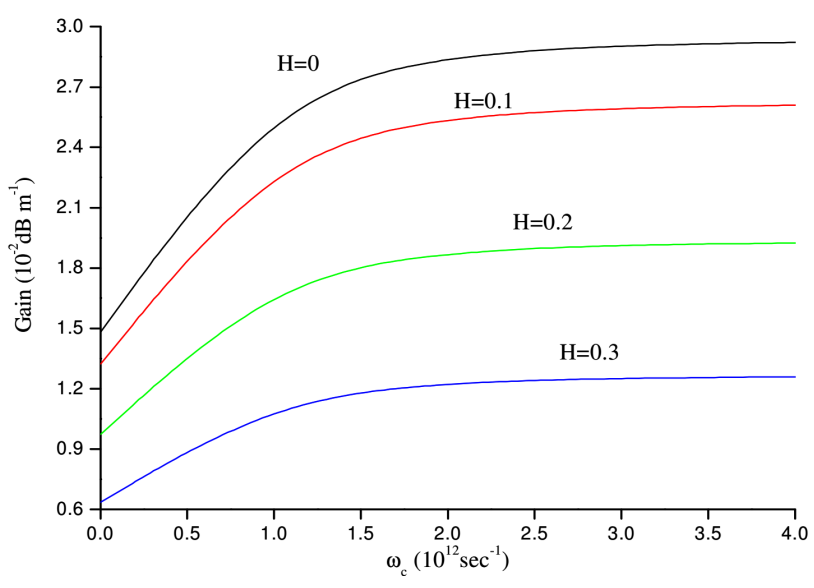

Fig. 1. $\frac{\alpha \omega}{\vartheta_{s}}$ versus $\omega_{c}$.

Figure 2 depicts the dependence of gain per radian $\alpha$ on velocity ratio $\frac{\vartheta_{0}}{\vartheta_{s}}$, for four different combinations of magnetic field and quantum parameter $H$. The nature of variation is found to be identical in each case i.e. magnitude of $\alpha$ has an increasing nature throughout the range of $\frac{\vartheta_{0}}{\vartheta_{s}}$ under study. Figure infers that the amplification $(\alpha>0)$ of acoustic wave is possible only when $\frac{\vartheta_{0}}{\vartheta_{s}}>1$.
Hence, one may very safely predict that the presence of magnetic field and quantum parameter $H$ does not influence the drift value, defining the crossover of the wave nature from attenuation to amplification. This fact is in concurrence with the experimentally obtained necessary condition of acoustic gain. The mechanism of such instability can be visualised as the Cerenkov production of phonons by fast electrons, moving in the piezoelectric medium, as the condition $\frac{\vartheta_{0}}{\vartheta_{s}}>1$ is analogous to the condition required for the Cerenkov radiation. The curves of Fig. 2 also show that the presence of magnetic field enhances the magnitude of gain, while quantum parameter$H$ reduces the gain, because its presence slows down the rate of energy exchange between plasmons and phonons.

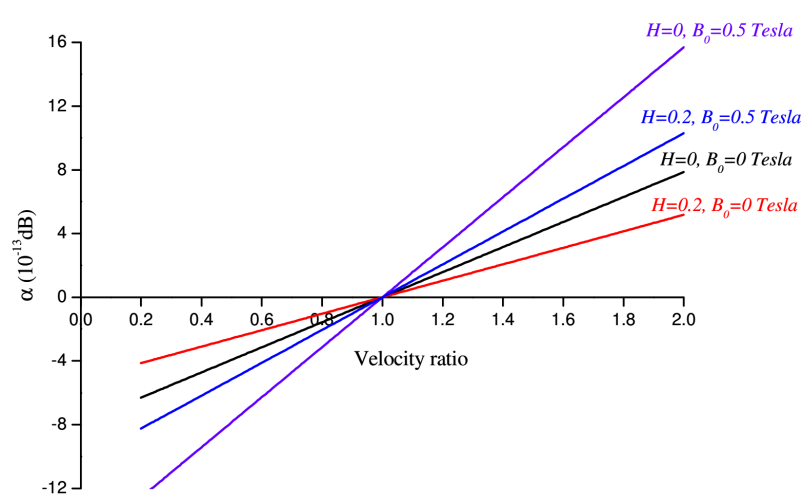

Fig. 2. $\alpha$ versus $\frac{\vartheta_{0}}{\vartheta_{s}}$.

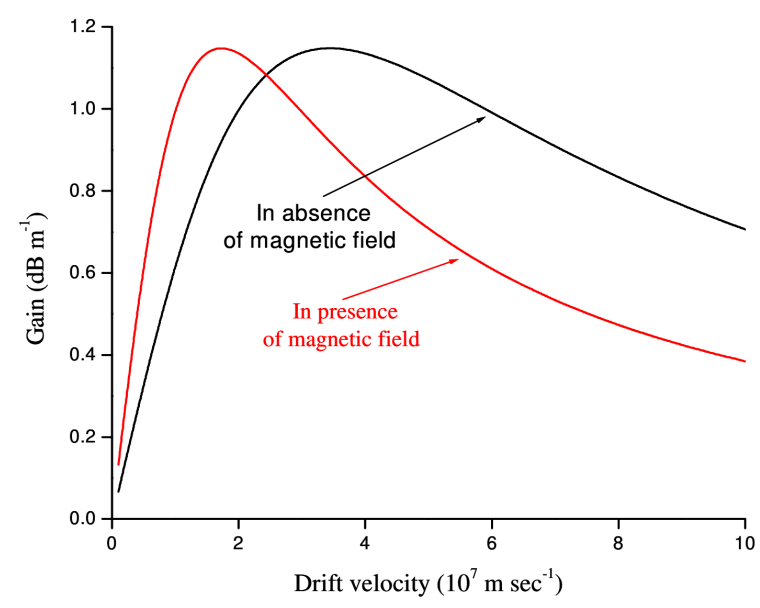

Fig. 3. $\frac{\alpha \omega}{\vartheta_{s}}$ versus $\vartheta_{0}$.

The influence of drifting of carriers $\left(\vartheta_{0}\right)$ on gain per unit length $\left(\frac{\alpha \omega}{\vartheta_{s}}\right)$ of acoustic wave, in magnetised $\left(B_{0} \neq 0\right)$ as well as unmagnetized $\left(B_{0}=0\right)$ semiconductor quantum plasma media is displayed in Fig. 3. One may deduct from this figure that in low velocity regime, $\frac{\alpha \omega}{\vartheta_{s}}$ rapidly increases with $\vartheta_{0}$, attains maxima and then starts decreasing as one goes towards high velocity regime. The carrier drift at which maximum gain occurs, may be defined as: 


$$
\vartheta_{0 c r} \approx \vartheta_{s}\left(1+\frac{\omega_{R} \omega_{D F}+\omega^{2}\left(1+\frac{k^{2} \vartheta_{\mathrm{F}}^{2}}{4 \omega_{p}^{2}} H^{2}\right)}{\omega \varphi \omega_{D F}}\right) .
$$

From this analytical expression of $\vartheta_{0 c r}$, it is clear that the presence of quantum correction term $H$ enhances the required magnitude of $\vartheta_{0 c r}$, at which maximum gain achieved. This figure also illustrates an important magnetic field effect on gain of acoustic wave through the parameter $\varphi$, that the presence of magnetic field shifts the maximum gain point towards lower drift velocity. This is in agreement with the analytical expression of $\vartheta_{0 \mathrm{cr}}$. Hence presence of magnetic field is found to be favourable for the amplification of acoustic wave in lower drift velocity regimes.

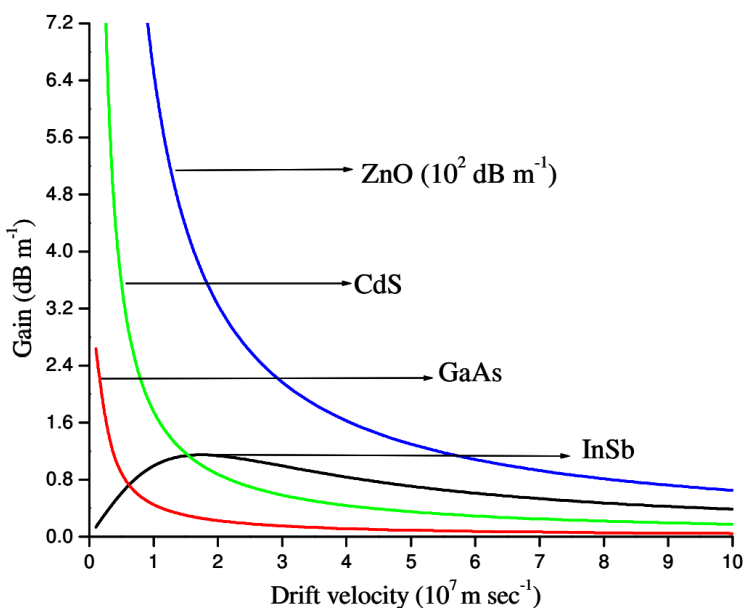

Fig. 4. $\frac{\alpha \omega}{\vartheta_{s}}$ versus $\vartheta_{0}$ for III-V and IV-VI semiconductors.

TABLE I

Acoustic behaviour of real materials

\begin{tabular}{c|c|c|c}
\hline \hline Semiconductors & $\begin{array}{c}\text { Piezoelectric } \\
\text { coefficient }(\bar{e}) \\
{\left[\mathrm{C} \mathrm{m}^{-2}\right]}\end{array}$ & Mobility & $\begin{array}{c}\left(\frac{\alpha \omega}{\vartheta_{s}}\right)_{\text {max }} \\
{\left[\mathrm{dB} \mathrm{m}^{-1}\right]}\end{array}$ \\
\hline $\mathrm{ZnO}$ & 1.32 & 0.02 & 720 \\
$\mathrm{CdS}$ & 0.21 & 0.035 & 7.2 \\
$\mathrm{GaAs}$ & 0.12 & 0.85 & 2.64 \\
$\mathrm{InSb}$ & 0.054 & 35.882 & 1.148
\end{tabular}

To get some idea about the parameter, based on which one may decide the material to be used, for acoustic device fabrication, we aimed to compare the acoustic behaviour of a few well known materials. We have studied the effect of drifting of carriers $\left(\vartheta_{0}\right)$ on gain per unit length $\left(\frac{\alpha \omega}{\vartheta_{s}}\right)$ of acoustic wave, in magnetised quantum plasma, for a few III-V and IV-VI semiconductors and the result is illustrated in Fig. 4. Figure infers that as $\vartheta_{0}$ increases, $\frac{\alpha \omega}{\vartheta_{s}}$ decreases for $\mathrm{ZnO}, \mathrm{CdS}$ and GaAs semiconductors, while $\frac{\alpha \omega}{\vartheta_{s}}$ increases initially, attains maxima, and then starts reducing with $\vartheta_{0}$ for InSb. The important inferences obtained from Fig. 4, is summarized in Table I.
From the tabulated values, it is hoped that a material with high piezoelectricity and low mobility is the best choice for acoustic device fabrication.

\section{Conclusion}

To conclude, we have examined the gain characteristics of acousto-electric interaction in piezoelectric semiconductor plasma, in presence of quantum parameter $H$ and magnetostatic field. Using macroscopic model of piezoelectricity and QHD model, we have derived dispersion relation and gain coefficient of acoustic wave in terms of quantum parameter $H$ and magnetic field. It has been observed that the presence of quantum parameter $H$ and magnetic field together play a crucial role in the modification of this interaction. Results show that application of magnetic field enhances the magnitude of gain whereas quantum effects, through quantum parameter $H$, reduce the gain of acoustic wave. But they do not affect the crossover point of wave nature from attenuation to amplification. Moreover, it has been also found that the presence of magnetic field shifts the maximum gain point towards lower drift velocity. Hence on the basis of the outcomes of this work, it turns out that gain profile of acoustic wave is very sensitive to change in magnitude of magnetic field and quantum parameter $H$. It may also be concluded that the results of our investigation may aid to understand the silent features of stability/instability regimes of acoustic wave in magnetised piezoelectric semiconductor quantum plasma.

\section{References}

[1] S. Ali, W.M. Moslem, P.K. Shukla, R. Schlickeiser, Phys. Plasmas 14, 082307 (2007).

[2] P.K. Shukla, B. Eliasson, Phys.-Usp. 53, 51 (2010).

[3] P.K. Shukla, B. Eliasson, Rev. Mod. Phys. 83, 885 (2011).

[4] I. Zeba, M.E. Yahia, P.K. Shukla, W.M. Moslem, Phys. Lett. A 376, 2309 (2012).

[5] M.R. Amin, Phys. Plasmas 22, 032303 (2015).

[6] K. Seeger, in: Semiconductor Physics, 9th ed., Springer, Berlin 2004.

[7] G. Manfredi, Fields Inst. Commun. 46, 263 (2005).

[8] A. Rasheed, M. Jamil, M. Siddique, F. Huda, Y.D. Jung, Phys. Plasmas 21, 062107 (2014).

[9] M. Marklund, P.K. Shukla, Rev. Mod. Phys. 78, 591 (2006).

[10] S. Ghosh, S. Dubey, R. Vanshpal, Phys. Lett. A 375, 43 (2010).

[11] M. Akbari-Moghanjoughi, Phys. Plasmas 18, 012701 (2011).

[12] S. Chandra, S.N. Paul, B. Ghosh, Ind. J. Pure Appl. Phys. 50, 314 (2012).

[13] F. Hass, A. Bret, Europhys. Lett. 97, 26001 (2012).

[14] S. Ghosh, S. Dubey, R. Vanshpal, Chin. J. Phys. 51, 1240 (2013).

[15] M.R. Amin, Astrophys. Space Sci. 359, 1 (2015).

[16] S.A. Maier, Plasmonics: Fundamentals and Applications, Springer, New York 2007. 
[17] N. Crouseilles, P.A. Hervieux, G. Manfredi, Phys. Rev. B 78, 155412 (2008).

[18] R. Vanshpal, S. Dubey, S. Ghosh, J. Phys. Confer. Ser. 365, 012045 (2012).

[19] Y. Wang, B. Eliasson, Phys. Rev. B 89, 205316 (2014).

[20] A. Rasheed, M. Jamil, F. Areeb, M. Siddique, M. Salimullah, J. Phys. D Appl. Phys. 49, 175109 (2016).

[21] J.S. Yang, H.G. Zhou, Acta Mech. 172, 113 (2004).

[22] S. Ghosh, P. Khare, Ind. J. Pure Appl. Phys. 44, 183 (2006).

[23] M. Willatzen, J. Christensen, Phys. Rev. B 89, 041201 (2014).
[24] V.K. Gokhale, M.R. Zadeh, Sci. Rep. 4, 5617 (2014).

[25] P. Li, F. Jin, J. Yang, Smart Mater. Struct. 24, 025021 (2015).

[26] A. Sharma, N. Nimje, N. Yadav, S. Ghosh, Int. J. Appl. Phys. 4, 37 (2014).

[27] P. Dubey, S. Ghosh, Acta Acust. United AC 102, 436 (2016).

[28] J. Christensen, M. Willatzen, V.R. Velasco, M.H. Lu, Phys. Rev. Lett. 116, 207601 (2016).

[29] M.C. Steele, B. Vural, Wave Interactions in Solid State Plasmas, McGraw Hill, New York 1969.

[30] D.L. White, J. Appl. Phys. 33, 2547 (1962). 